\title{
MAPKK Inhibitor U0126 Inhibits Plasmodiophora brassicae Development
}

\author{
Tao Chen, Kai Bi, Yanli Zhao, Xueliang Lyu, Zhixiao Gao, Ying Zhao, Yanping Fu, Jiasen Cheng, \\ Jiatao Xie, and Daohong Jiang ${ }^{\dagger}$
}

State Key Laboratory of Agricultural Microbiology, and The Provincial Key Lab of Plant Pathology of Hubei Province, College of Plant Science and Technology, Huazhong Agricultural University, Wuhan, 430070, Hubei Province, PR China.

Accepted for publication 25 January 2018.

\begin{abstract}
Mitogen-activated protein kinase (MAPK) cascades play a central role in cellular growth, proliferation, and survival. MAPK cascade genes have been extensively investigated in model plants, mammals, yeast, and fungi but are not characterized in Plasmodiophora brassicae, which causes clubroot disease in cruciferous plants. Here, we identified 7 PbMAPK, 3 PbMAPKK, and 9 PbMAPKKK genes in the P. brassicae genome. Transcriptional profiling analysis demonstrated that several MAPK, MAPK kinase (MAPKK), and MAPK kinase kinase (MAPKKK) genes were preferentially expressed in three different

PbMAK3. The PbMAKKK7-PbMAKK3-PbMAK1/PbMAK3 cascade may be present in $P$. brassicae. U0126, a potent and specific inhibitor of MAPKK, could inhibit the germination of $P$. brassicae resting spores. U0126 was used to treat the resting spores of $P$. brassicae and coinoculate rapeseed, and was proven to significantly relieve the severity of clubroot symptoms in the host plant and delay the life cycle of $P$. brassicae. These results suggest that MAPK signaling pathways may play important roles in $P$. brassicae growth, development, and pathogenicity.
\end{abstract} zoosporic stages. Based on yeast two-hybrid assays, PbMAKKK 7 interacted with PbMAKK3 and PbMAKK3 interacted with PbMAK1/

Plasmodiophora brassicae, which causes clubroot disease in cruciferous plants, is an emerging threat to the production of Brassica oil and vegetable crops (Braselton 1995; Hwang et al. 2012). Clubroot occurs in more than 60 countries worldwide, and is more prevalent in those areas where Brassica crops are intensively cultivated, such as Europe, Australia, and North America (Dixon 2009). Clubroot disease is characterized by the formation of clubshaped galls on the roots of infected plants. P. brassicae is a soilborne and obligate biotroph protist that resides entirely inside the host plant cell. Furthermore, the resting spores (RS) of $P$. brassicae can survive in the soil for up to 20 years, making it very difficult to manage clubroot (Dixon 2009). Despite its agricultural importance, the pathogenicity of $P$. brassicae remains poorly understood, especially at the molecular level. $P$. brassicae has a complex and not completely understood infection process. RS release primary zoospores and form primary plasmodia inside root hairs. After a number of nuclear divisions, the plasmodia cleave into zoosporangia, and the zoosporangia form clusters in the root hair or penetrate into root cortex cells, in which the pathogen develops into secondary plasmodia and, finally, into a new generation of RS (Kageyama and Asano 2009). The genomes of P. brassicae have some typical biotrophic features of eukaryotic plant pathogens (Schwelm et al. 2015). Both P. brassicae pathotypes 3 and 6 exhibit highly compact genomes, dependency on its host for some essential nutrients, and a potential role in the regulation of host plant

${ }^{\dagger}$ Corresponding author: D. Jiang; E-mail: daohongjiang@mail.hzau.edu.cn

Funding: This work was supported by the earmarked fund for China Agriculture Research System (CARS-13) and by the Fundamental Research Funds for the Central Universities (program number 2662017JC004)

This research was conducted in the absence of any commercial or financial relationships that could be construed as a potential conflict of interest.

*The $\boldsymbol{e}$-Xtra logo stands for "electronic extra" and indicates that five supplementary figures and two supplementary tables are published online.

(c) 2018 The American Phytopathological Society
Additional keywords: MAPKK inhibitor U0126.

cytokinin and auxin (Rolfe et al. 2016). It was recently reported that lipid droplets of $P$. brassicae are an important cellular organelle that stores neutral lipids. Genome, transcriptome, and proteome studies have been integrated to investigate the lipid synthesis, storage, and metabolism in $P$. brassicae (Bi et al. 2016). Nevertheless, it remains challenging to elucidate the functions of proteins in these uncultivable protists.

Mitogen-activated protein kinases (MAPK) play pivotal roles in signal transduction and govern diverse and critical cellular processes, including proliferation, differentiation, and cell survival in all eukaryotes (Aouadi et al. 2006; Ichimura et al. 2002; Xia et al. 1995; Zhang and Liu 2002). Generally, a MAPK pathway consists of three classes of protein kinases: MAPK (MPK), MAPK kinase (MAPKK/MKK/MEK), and MAPK kinase kinase (MAPKKK/ MEKK). MAPK are phosphorylated and activated by MAPKK, which themselves are activated by MAPKKK (Chang and Karin 2001; Katz et al. 2007). The first signaling module identified in plants is the Arabidopsis MEKK1-MKK4/5-MPK3/6 cascade, which plays a vital role in plant innate immunity (Asai et al. 2002). This cascade also participates in stomatal development and dynamics (Wang et al. 2007). The Arabidopsis MEKK1-MKK1/2-MPK4 cascade was shown to positively regulate defense responses against necrotrophic fungi while negatively regulating defense against biotrophic pathogens (Gao et al. 2008). The best characterized MAPK cascade is the yeast Ste11-Ste7-Fus3/Kss1 pathway involved in pheromone response and filamentous growth (Chen and Thorner 2007). Its orthologs have been shown to regulate different infection processes in dozens of plant-pathogenic fungi (Hamel et al. 2012; Meng and Zhang 2013). Although MAPKK pathways have been well characterized in a number of plants and pathogenic fungi, no systematic and genome-wide investigations of the MAPK signaling cascade gene families have been reported in $P$. brassicae yet.

In the present study, seven $P b M A P K$, three $P b M A P K K$, and nine $P b M A P K K K$ genes were identified in $P$. brassicae, which were then validated by comparison with Arabidopsis genes AtMAPK, AtMAPKK, and AtMAPKKK based on phylogenetic analyses. Transcriptome analysis of these $P$. brassicae MAPK proteins during three different zoosporic stages demonstrated their important roles 
in the regulation of $P$. brassicae growth and development. The yeast two-hybrid assay showed that a PbMAPKKK7-PbMAPKK3-PbMAPK1/ $P b M A P K 3$ cascade may be present in $P$. brassicae. We investigated the effects of a specific inhibitor of MAPKK on the growth and development of $P$. brassicae, and found that MAPKK inhibitor U0126 could inhibit the germination of RS, relieve the clubroot symptoms, reduce the pathogenicity of $P$. brassicae, and decrease the growth and development of $P$. brassicae, suggesting that $P b M A P K K$ play important roles in the growth and development of $P$. brassicae.

\section{MATERIALS AND METHODS}

Identification of MAPK, MAPKK, and MAPKKK gene families in $P$. brassicae. The genome of $P$. brassicae was downloaded from DDBJ/ENA/GenBank under accession number MCBL 01000000 (Bi et al. 2016). MAPK, MAPKK, and MAPKKK gene families were queried using the National Center for Biotechnology Information (NCBI) Conserved Domain Database (CDD) (https:// www.ncbi.nlm.nih.gov/Structure/cdd/wrpsb.cgi) and batch CD-Search tool, which facilitates the processing of up to 100,000 protein queries at a time (Marchler-Bauer et al. 2011). All data were checked for redundancy by self-BLAST and no alternative splice variants were considered. Only the MAPKKK genes that displayed consensus sequences GTXXWMAPE were accepted. MAPKK genes were accepted only if they contained the conserved sequences $\mathrm{D}(\mathrm{L} / \mathrm{I} / \mathrm{V}) \mathrm{K}$ and signature VGTXxYMSPER, whereas MAPK genes should contain the TXY motif. Then, the NCBI CDD (https://blast.ncbi.nlm.nih.gov/) and SMART databases (http://smart.embl-heidelberg.de/) were used to further confirm the predicted MAPK cascade genes. The isoelectric point (pI) and molecular weight of the obtained proteins were predicted using the Compute pI/Mw tool online (http://web.expasy.org/compute_pi/). Subcellular localization prediction of each protein was performed by CELLO v.2.5 online (http://cello.life.nctu.edu.tw/) (Yu et al. 2006).

Multiple sequence alignment, phylogenetic analysis, and gene structure construction. Multiple sequence alignments were performed using DNAMAN or ClustalX2. The online MEME software (http://meme-suite.org/tools/meme) was used for domain and motif detection (Bailey et al. 2006). Phylogenetic analysis was performed based on the full-length protein sequences or conserved domain using the MEGA 6 program by the neighbor-joining method, and a bootstrap test was carried out with 1,000 interactions (Saitou and Nei 1987).

Plant materials, growth conditions, and U0126 inhibitor treatment. Brassica napus 'Hua shuang 4' was surface sterilized with household sodium hypochlorite and pregerminated on a sterilized wet filter paper in a Petri dish, then grown in a 10-ml tubes with halfstrength $(1 / 2)$ Hoagland nutrient solution. The plants were grown in a plant growth chamber maintained at $70 \%$ humidity and $23^{\circ} \mathrm{C}$ with a cycle of $16 \mathrm{~h} /$ day and $8 \mathrm{~h} /$ night. Ten-day-old seedlings were inoculated with $1 \mathrm{ml}$ of RS of $P$. brassicae strain $\mathrm{ZJ}-1\left(1 \times 10^{7}\right.$ spores $\left./ \mathrm{ml}\right)$, originally isolated from a diseased plant in a rapeseed field in Zhijiang County, Hubei Province, PR China (Bi et al. 2016; Chen et al. 2016). For U0126 inhibitor treatment, a stock solution of U0126 was prepared in dimethyl sulfoxide (DMSO). U0126 is an elective inhibitor of MEK-1 and MEK-2 and a weak inhibitor of protein kinases such as PKC, Raf, ERK, JNK, MEKK, MKK-3, MKK-4/SEK, MKK-6, Abl, Cdk2, and Cdk4 (Favata et al. 1998). Inhibitor U0126 (MedChemExpress) at a concentration of 50 or $100 \mathrm{nM}$ was added to the $1 / 2$ Hoagland nutrient solution during inoculation. We filled the tubes with $1 / 2$ Hoagland nutrient solution every day, and completely changed the 1/2 Hoagland nutrient solution each 7 days after inoculation to avoid contamination.

Disease index quantification of MAPKK U0126 inhibitor treatment. Disease index was calculated at 35 days postinoculation (dpi). Disease severity was assessed using a scoring system of 0 to 4 , as previously reported (Siemens et al. 2002). A score of 0 indicated no disease; 1 = very small galls, mainly on lateral roots that did not impair the main root; 2 = small galls covering the main root and a few lateral roots; 3 = medium to large galls on both lateral roots and main root; and 4 = severe galls on lateral root, main root, or rosette, with fine roots completely destroyed. Disease index was calculated using the five-grade scale according to the formula Disease index $=\left(1 n_{1}+\right.$ $\left.2 n_{2}+3 n_{3}+4 n_{4}\right) \times 100 / 4 \mathrm{~N}_{\mathrm{t}}$, where $n_{1}$ to $n_{4}$ is the number of plants in the indicated class and $N_{\mathrm{t}}$ is the total number of plants tested. For each biological experiment, at least 22 plants were analyzed. Similar results were derived from three independent biological experiments.

DNA extraction and quantitative polymerase chain reaction expression analysis. DNA was extracted from root samples using the cetyl trimethyl ammonium bromide method (Allen et al. 2006). Quantitative polymerase chain reaction (PCR) was performed on a CFX96 real-time PCR system (Bio-Rad) using iTaq Universal SYBR Green supermix (Bio-Rad) to quantify the $P$. brassicae target actin gene AY452179.1. Each reaction was performed with $2.5 \mathrm{ng}$ of total DNA as template, and B. napus actin gene AF111812 was used as an internal control for data normalization. Standard curves were constructed using serial dilutions of DNA extracted from the roots of B. napus at $35 \mathrm{dpi}$ with $P$. brassicae, which was defined as a reference condition. Quantitative results were then expressed as percentage of the $P$. brassicae mean DNA content in this reference condition (Chen et al. 2016). The following primers were used for gene expression analysis: 5' -CACCGACTACCTGATGAA-3' and 5'-CAGCTTCTCCTTGATGTC-3' for Pbactin and 5'-TGAAG ATCAAGGTGGTCGCA-3' and 5'-AGAAGGCAGAAACACTTAG AAG-3' for Bnactin.

cDNA cloning, plasmid constructions and yeast twohybrid assays. Two MAPK, one MAPKK, and one MAPKKK genes were selected from the KEGG pathway of the MAPK signaling pathway in P. brassicae. A complete list of the primers used for reversetranscription PCR, cDNA cloning, and expression studies is presented in Supplementary Table S1. The amplified PbMAPK1 full-length cDNA was cloned into the $N d e \mathrm{I} /$ Bam HI sites of pGADT7, the amplified $P b M A P K 3$ full-length cDNA was cloned into the NdeI/EcoRI sites of pGADT7, and the full-length cDNA encoding the PbMAPKKK7 was amplified by PCR and cloned into EcoRI/SmaI sites of pGADT7. The amplified PbMAPKK3 full-length cDNA was cloned into the EcoRI/ PstI sites of pGBKT7. Constructs of pGBKT7 were transformed into yeast strain Y187 by the lithium acetate method and constructs of pGADT7 were transformed into yeast strain AH109. Yeast Y187 cells expressing a Gal4 DNA-binding domain fusion protein were mated with yeast AH109 cells harboring a Gal4 activation domain fusion construct. The diploid cells were selected on SD media lacking Leu and Trp, and tested for protein-protein interactions on selective SD media lacking Leu, Trp, His, and Ade. SD is a minimal defined medium containing (per liter) $6.7 \mathrm{~g}$ of yeast nitrogen base without amino acids (Difco), $20 \mathrm{~g}$ of agar (for plates only), $100 \mathrm{ml}$ of the appropriate sterile $10 \times$ Dropout Solution, and $850 \mathrm{ml}$ of $\mathrm{H}_{2} \mathrm{O}$. The interaction between mammalian p53 and SV40 served as a positive control, whereas coexpression of lamin and SV40 served as a negative control.

Availability of supporting data. The Whole Genome Shotgun project has been deposited at DDBJ/ENA/GenBank under the accession number of MCBL00000000. The version described in this article is MCBL01000000. The expression data sets used in this study are available at the NCBI Gene Expression Omnibus (https://www.ncbi.nlm.nih. gov/geo/) under accession number SRP079943 (Bi et al. 2016).

Statistical analysis. Statistical analysis was performed using SAS (8.1) statistics software. All experiments were repeated independently three times. Data obtained were subjected to statistical analysis using Student's $t$ tests and probability values of $P<0.05$ were considered as significant between different treatments.

Scanning electron microscopy. Infected roots were harvested and washed with distilled water. Sections of 1 to $2 \mathrm{~mm}$ were cut from the clubs. The samples were fixed in $2 \%$ glutaraldehyde, dehydrated in a graded ethanol series $(30,50,70,80,90$, and $100 \%)$, and then placed on a carbon grid and sputter coated for $5 \mathrm{~min}$ with gold. Examinations were performed with a HITACHI SU-8010 scanning electron microscope.

Germination assay of $\boldsymbol{P}$. brassicae RS. The effect of MAPKK inhibitor U0126 on the germination of $P$. brassicae RS was assessed in the presence of host root exudates ( $B$. rapa seedlings) 
according to the previously described method (Jäschke et al. 2010; Niwa et al. 2008). Seed of cultivar Hua shuang 4 were surface sterilized with household sodium hypochlorite and pregerminated on a wet filter paper in Petri dishes. After a week, root exudates were extracted by using a bacterial filter. RS of $P$. brassicae $\left(10^{7}\right.$ spores $\left./ \mathrm{ml}\right)$ with 50 or $100 \mathrm{nM}$ inhibitor U0126 were incubated for 3 or 6 days in root exudates. DMSO was used instead of inhibitor as a negative control. The volume of filtrate was reduced after centrifugation, achieving a final spore density of 5,000 to 10,000 spores. A $100-\mu$ l aliquot of the spore suspension was mixed and stained with an equal volume of $4^{\prime}-6-$ diamidino-2-phenylindole-dihydrochloride (DAPI; Sigma-Aldrich) at $2 \mu \mathrm{g} / \mathrm{ml}$ in $0.1 \%$ ( $\mathrm{vol} / \mathrm{vol}$ ) dimethylformamide, and $5 \mu \mathrm{l}$ of the mixture was transferred onto a glass slide and covered with an 18-by18 -mm cover glass. The total numbers of spores and the number of spores with a nucleus in one field of view were counted under UV excitation, using a fluorescent microscope at $\times 400$ magnification. Approximately 200 spores were examined from each sample and each sample was repeated three times.

\section{RESULTS}

Identification of MAPK, MAPKK, and MAPKKK families in P. brassicae. Availability of complete P. brassicae genome sequences for the first time makes it possible to identify all of the MAPK, MAPKK, and MAPKKK family members in this obligate biotroph protist species. The three family genes were determined by using the NCBI CDD and batch CD-Search tool, which facilitates the processing of up to 100,000 protein queries at a time (Marchler-Bauer et al. 2011). All potential MAPK cascade sequences contained the serine/threonine-protein kinase-like domain (superfamily of cl121453) in $P$. brassicae, resulting in a total of 214 hits. Sequences that did not contain the known conserved motifs of MAPK, MAPKK, or MAPKKK family proteins were excluded from further BLASTP analysis. After multiple steps of screening and validation of the conserved domains, we identified seven PbMAPK, three PbMAPKK, and nine PbMAPKKK genes. The total number (19) was obviously smaller than that in Arabidopsis thaliana (110) (Ichimura et al. 2002; Jonak et al. 2002) and Oryza sativa (100) (Rao et al. 2010; Singh et al. 2012) but was close to that in Caenorhabditis elegans (22) (Andrusiak and Jin 2016; Plowman et al. 1999) and larger than that in the fungus Saccharomyces cerevisiae (14) (Hamel et al. 2012) and plant-pathogenic fungi Magnaporthe oryzae (10), Botrytis cinerea (10), and Ustilago maydis (12) (Hamel et al. 2012) (Supplementary Table S2). Detailed information about the three gene family members, including gene identification, accession number, number of deduced amino acid residues, molecular weight, $\mathrm{pI}$, and predicated subcellular localization, is shown in Table 1 . These PbMAPK genes were predicted to encode 385 to 509 amino acids in length, with putative molecular weights of 43.0 to $56.8 \mathrm{kDa}$ and $\mathrm{pI}$ of 6.3 to 10.1 . The subcellular localization was predicated to be in the mitochondria, cytoplasma, or nuclei (Table 1). Meanwhile, the three predicted PbMAPKK proteins contained 352 to 405 amino acids, with molecular weights of 38.9 to $44.8 \mathrm{kDa}$ and $\mathrm{pI}$ of 6.03 to 9.09 . They were predicted to be localized in the mitochondria or nuclei (Table 1). The polypeptide lengths of the nine PbMAPKKK genes ranged from 353 to 1,303 amino acids, with molecular weights of 39.2 to $145.5 \mathrm{kDa}$ and pI of 5.12 to 9.27. PbMAPKKK proteins were located in mitochondria, cytoplasm, nuclei, or plasma membranes (Table 1).

Multiple sequence alignment and motif analysis. Multiple sequence alignment of the predicted amino acid residues was conducted on the PbMAPK gene family. The data showed that all PbMAPK contained a classical TXY motif, which is located in the activation loop between the subdomains VII and VIII of MAPK (Ichimura et al. 2002). The conserved motifs of the seven PbMAPK proteins were also analyzed with the online tool MEME. Thus, a schematic representation of the motifs is provided in Supplementary Fig. S1. Eight motifs were conserved in all of the PbMAPK proteins. Multiple sequence alignment of the PbMAPKK proteins showed that each of them contained the conserved lysine $(\mathrm{K})$ and aspartate $(\mathrm{D})$ residues within the active site motif D (L/I/V) K, and signature VGTxxYMSPER, which was conserved in the catalytic domain (Supplementary Fig. S2). Plant MAPKK have the consensus sequence S/TxxxxxS/T, whereas mammalian enzymes have S/TxxxS/T in the activation loop (MAPK Group et al. 2002). However, this consensus sequence was not found in PbMAPKK, and similar results were observed in AtMAPKK10, OsMAPKK10-1, OsMAPKK10-2, and OsMAPKK10-3 (Wang et al. 2015). Six of the eight motifs were conserved in all of the PbMAPKK proteins, whereas motif 7 and 8 were group specific. Motif 1 had the signature of VGTxxYMSPER, which was conserved in the catalytic domain; motif 3 had an active-site signature HRDxKPsNILL of serine/ threonine protein kinases, and motif 6 contained the protein kinase ATPbinding signature that requires a glycine-rich loop (GxGxxG) for ATP binding. As reported in Arabidopsis and other species, MAPKKK were classified into three subfamilies, including MEKK (with conserved signature $\mathrm{G}(\mathrm{T} / \mathrm{S}) \mathrm{Px}(\mathrm{W} / \mathrm{Y} / \mathrm{F}) \mathrm{MAPEV})$, Raf (with conserved signature GTxx(W/Y)MAPE) and ZIK (with conserved signature GTPEFMAPE (L/V)Y) (Wang et al. 2015). However, we only found nine Raf-like MAPKKK in P. brassicae (Supplementary Fig. S3). Four of the eight motifs were conserved in all of the PbMAPKKK proteins. Motif

TABLE 1. Characteristics of the mitogen-activated protein kinase (MAPK), MAPK kinase (MAPKK), and MAPK kinase kinase (MAPKKK) genes in Plasmodiophora brassicae ${ }^{\mathrm{a}}$

\begin{tabular}{|c|c|c|c|c|c|c|}
\hline Name & Gene ID & Accession number & Length (aa) & MW (kDa) & pI & Subcellular location \\
\hline PbMAPK1 & PlasB_00238 & KY776455 & 398 & 44.9 & 6.5 & Cytoplasma, nuclei \\
\hline PbMAPK2 & PlasB_03290 & KY776456 & 509 & 56.8 & 8.5 & Nuclei, mitochondria \\
\hline PbMAPK3 & PlasB_02211 & KY776457 & 401 & 44.6 & 6.3 & Mitochondria, cytoplasma \\
\hline PbMAPK4 & PlasB_07916 & KY776458 & 385 & 43.0 & 6.8 & Nuclei, cytoplasma \\
\hline PbMAPK5 & PlasB_09030 & KY776459 & 393 & 44.7 & 9.7 & Nuclei, cytoplasma \\
\hline РbMAРК6 & PlasB_00298 & KY776460 & 475 & 52.7 & 10.1 & Mitochondria, nuclei \\
\hline PbMAPK7 & PlasB_06370 & KY820693 & 416 & 47.5 & 8.8 & Mitochondria \\
\hline PbMAPKK1 & PlasB_04173 & KY776461 & 405 & 44.8 & 9.1 & Nuclei \\
\hline$P b M A P K K 2$ & PlasB_06889 & KY776462 & 392 & 43.6 & 8.4 & Mitochondria \\
\hline$P b M A P K K 3$ & PlasB_03812 & KY776463 & 352 & 38.9 & 6.0 & Extracellular, nuclei \\
\hline PbMAPKKK1 & PlasB_06871 & KY776464 & 1,126 & 125.1 & 9.3 & Nuclei, plasma membrane \\
\hline PbMAPKKK2 & PlasB_00670 & KY776465 & 353 & 39.2 & 9.7 & Mitochondria \\
\hline PbMAPKKKЗ & PlasB_00843 & KY776466 & 765 & 86.0 & 6.5 & Plasma membrane, nuclei \\
\hline PbMAPKKK 4 & PlasB_07093 & KY776467 & 1,303 & 145.5 & 7.8 & Nuclei \\
\hline PbMAPKKK5 & PlasB_07100 & KY776468 & 1,090 & 119.8 & 5.1 & Cytoplasma \\
\hline PbMAРКKК6 & PlasB_09434 & KY776469 & 969 & 106.8 & 7.2 & Cytoplasma, mitochondria \\
\hline PbMAPKKK 7 & PlasB_03097 & KY820691 & 752 & 82.8 & 8.1 & Nuclei \\
\hline PbMAPKKK & PlasB_06795 & KY820692 & 420 & 45.9 & 5.60 & Cytoplasma \\
\hline PbMAРКKKУ & PlasB_00740 & KY820694 & 1,094 & 121.9 & 5.8 & Cytoplasma, nuclei \\
\hline
\end{tabular}

a Abbreviations: $\mathrm{aa}=$ amino acids, $\mathrm{MW}=$ molecular weight, and $\mathrm{pI}=$ isoelectric point. 
annotation showed that motif 6 contained a protein kinase ATP-binding site, whereas motif 1 contained the serine/threonine protein kinase active site. To further explore the evolutionary relationships of the three gene families in P. brassicae and in A. thaliana, we selected five MAPK, five MAPKK, and eight MAPKKK from A. thaliana, and seven MAPK, three MAPKK, and nine MAPKKK from $P$. brassicae to construct a phylogenetic tree. As expected, $P b M A P K, P b M A P K K$, and $P b M A P K K K$ could be grouped with their counterparts in Arabidopsis (Fig. 1).

Expression profiles in different developmental stages. There has been increasing evidence showing that MAPK cascade genes are widely involved in the growth and development of protists. Here, we identified MAPK gene families and compared the wholegenome transcriptomes of typical developmental stages of $P$. brassicae: purified mature RS, germinating spores exposed to host root exudates (GS), and secondary plasmodia during the cortex infection (CI) stage (Bi et al. 2016). The expression levels of these genes and samples were clustered and presented in heatmaps (Fig. 2). Transcriptome analysis of PbMAPK, PbMAPKK, and PbMAPKKK showed that the expression level of the RS sample was similar to that of the GS sample but differed from that of CI sample (Fig. 2), suggesting that MAPK cascade proteins have a greater effect on CI samples than on GS samples. Except for PbMAPK2, all PbMAPK and PbMAPKK genes were observed to be upregulated in the CI stage, and may mainly function in the proliferation of secondary plasmodia (Fig. 2). PbMAPK6 and PbMAPKKK8 showed very low expression in all of the tested development stages, whereas all of the MAPK cascade members were expressed in at least one developmental stage. Several proteins showed striking differences in expression levels among different development stages (Fig. 2). Except for PbMAPKKK4 and $P b M A P K K K 8$, all $P b M A P K K K$ genes had relatively high expression levels in the three different developmental stages (Fig. 2). Therefore, these genes may mainly function in the development of P. brassicae.

Interactions between PbMAPKKK7 and PbMAPKK3, and $P b M A P K K 3$ and $P b M A P K$, in yeast. MAPK cascades are conserved signaling modules present in all eukaryotic cells, including plants, fungi, and animals. However, only a few Arabidopsis MAPK cascade components and their interactions have been studied and

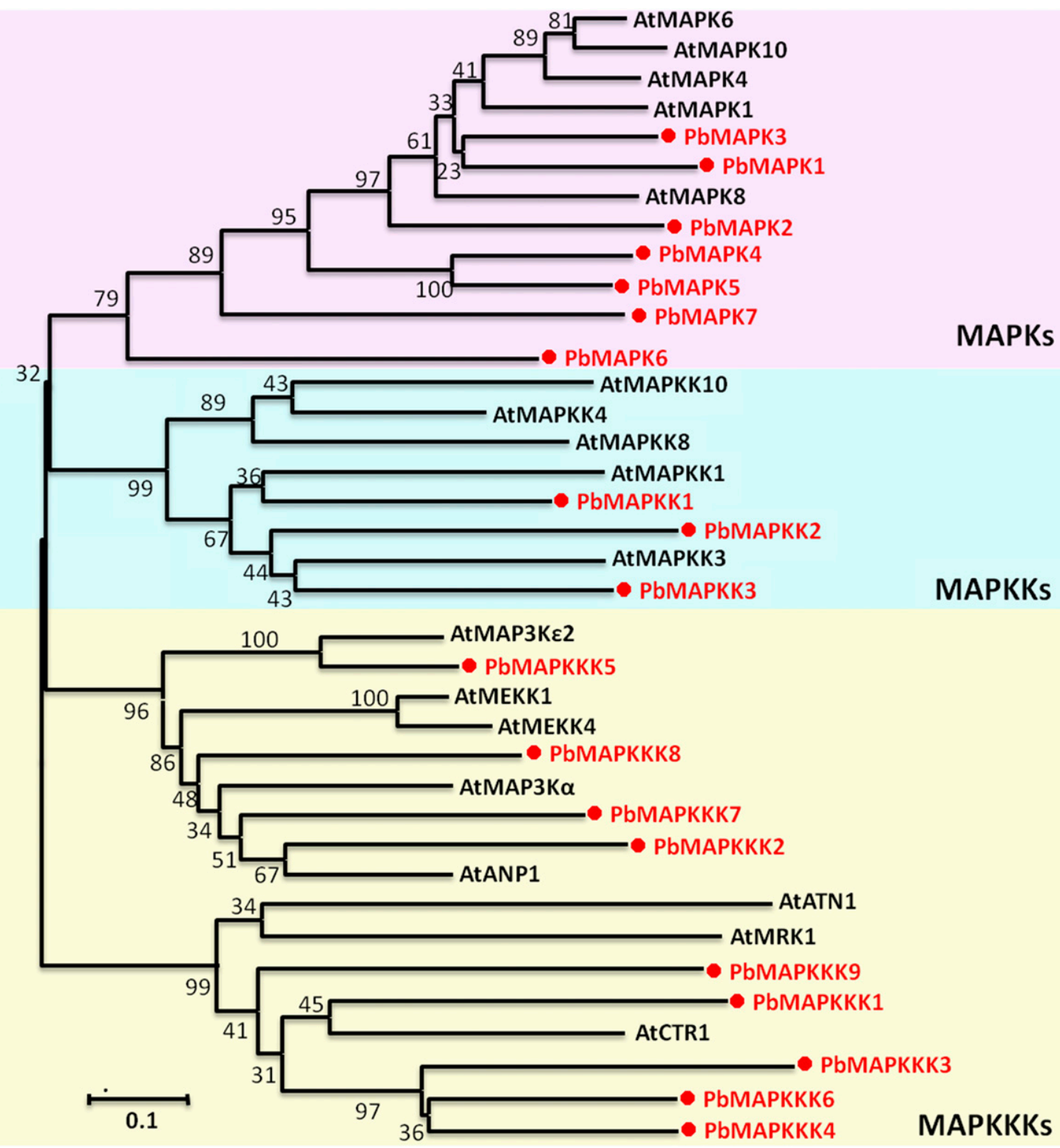

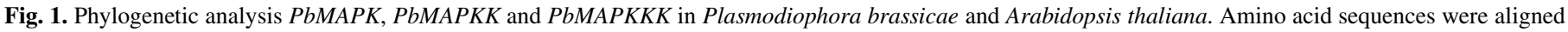

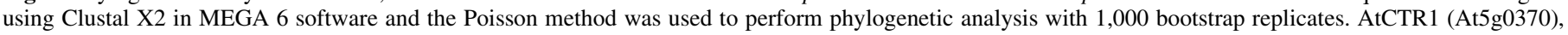

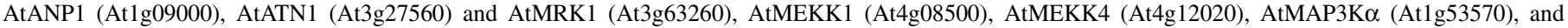
AtMAP3Ke2 (At3g07980) are typical mitogen-activated protein kinase kinase kinases (MAPKKK). 
reported in detail thus far. In a typical MAPK cascade, a MAPKKK activates specific MAPKK, and a MAPKK activates specific MAPK. In order to unravel the MAPKKK-MAPKK and MAPKK-MAPK interactions in $P$. brassicae, we selected some MAPK cascade components from the MAPK signaling pathway in the KEGG pathway categories of $P$. brassicae genome. The results of yeast two-hybrid assays were shown in Figure 3. PbMAPKKK7 could interact with $P b M A P K K 3$; and $P b M A P K K 3$ could interact with both $P b M A P K 1$ and $P b M A P K 3$. These results suggested the presence of a PbMAPKKK7PbMAPKK3-PbMAPK1/PbMAPK3 MAPK cascade in $P$. brassicae.

MAPKK inhibitor U0126 treatment inhibited $P$. brassicae $\mathrm{RS}$ germination. To examine the inhibitory effects of MAPKK on RS germination, RS were treated with $50 \mathrm{nM}$ (inhibitor-treated group A) or $100 \mathrm{nM}$ (inhibitor-treated group B) U0126 and collected 3 or 6 days later for nuclear status examination with DAPI. Detection of ungerminated spores retaining one zoospore (with a nucleus) is a reliable assay system to examine the germination of RS (Niwa et al. 2008). The absence or presence of a nucleus in the RS could be discriminated under UV excitation (Fig. 4A). After 3 days of incubation, in the two U0126 treatment experiments, the mean percentages of $P$. brassicae spores without a nucleus were 42 and $43 \%$, respectively, whereas the percentage was $73 \%$ in the control group (Fig. 4B). Similar results were observed after 6 days of incubation. The germination rates of the RS in MAPKK inhibitor groups were 36 and $41 \%$, respectively, and $78 \%$ for the control group (Fig. 4A and B). Based on these results, it could be suggested that germination of $P$. brassicae RS was significantly inhibited by MAPKK inhibitor treatment.

MAPKK inhibitor U0126 treatment reduced pathogenicity of $P$. brassicae. MAPK signaling pathways have been

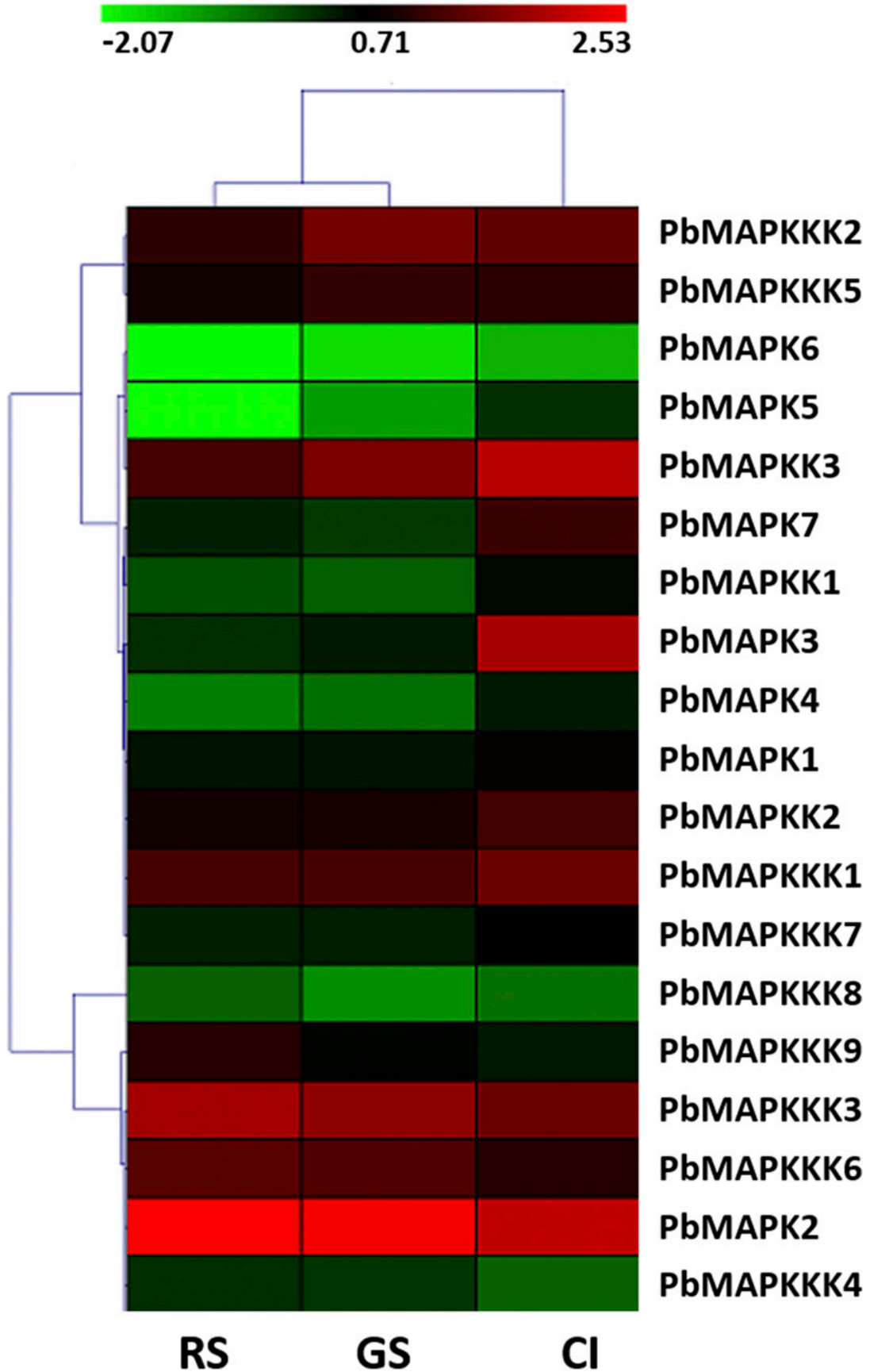

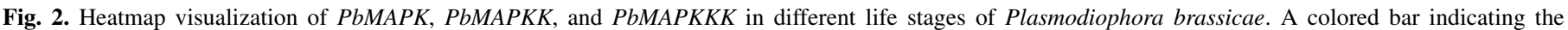

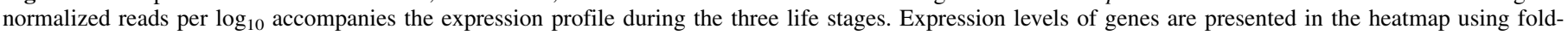

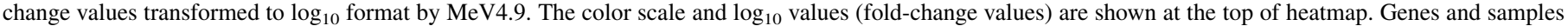
were clustered according to their expression profiles. RS = resting spores, GS = germinating spores, and CI = cortex infection. 
demonstrated to be critical for cell differentiation. To further characterize the biological functions of MAPK cascades, we analyzed their responses to MAPKK-specific inhibitor U0126 treatment. Host plants were grown in liquid nutrient solution and coinoculated with RS of $P$. brassicae and 50 or $100 \mathrm{nM}$ MAPKK inhibitor U0126. The formation of root galls was assessed at $35 \mathrm{dpi}$. The clubroot symptom of the infected group without inhibitor treatment was more severe than that of the inhibitor-treated groups: the former showed galls on the roots whereas the latter did not form obvious galls on the roots (Fig. 5A). In addition, no obvious differences were observed in the leaves of the four groups (Supplementary Fig. S4), suggesting that the concentrations of U0126 used in this study were not toxic for the host cells. We then evaluated the disease index in plants treated with and without inhibitor (Fig. 5B). The disease index was 87.5 in the infected
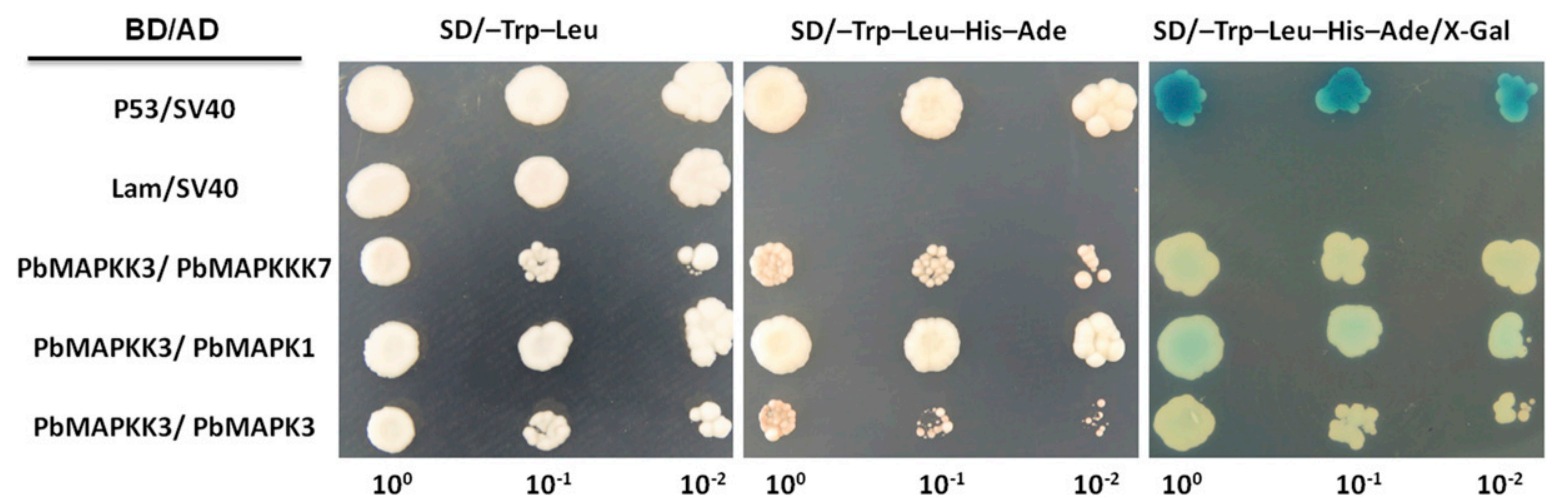

Fig. 3. Interaction between selected PbMAPKKK with PbMAPKK, and PbMAPKK with PbMAPK, in yeast. Proteins were fused with the Gal4 DNA-binding domain (BD) in pGBKT7 or with its activation domain (AD) in pGADT7. Yeast cells harboring the constructs were maintained on SD/-Trp-Leu medium (SD-2) and selected for protein-protein interaction on SD/-Trp-Leu-His-Ade (SD-4) or SD/-Trp-Leu/X-gal (SD-2/X-gal). The interaction between murine tumorsuppressor p53 and simian virus 40 large T-antigen (pGBKT7-53 and pGADT7-SV40) was used as a positive control, whereas the combination between human lamin C (Lam) and SV40 (pGBKT7-Lam and pGADT7-SV40) served as a negative control.

A
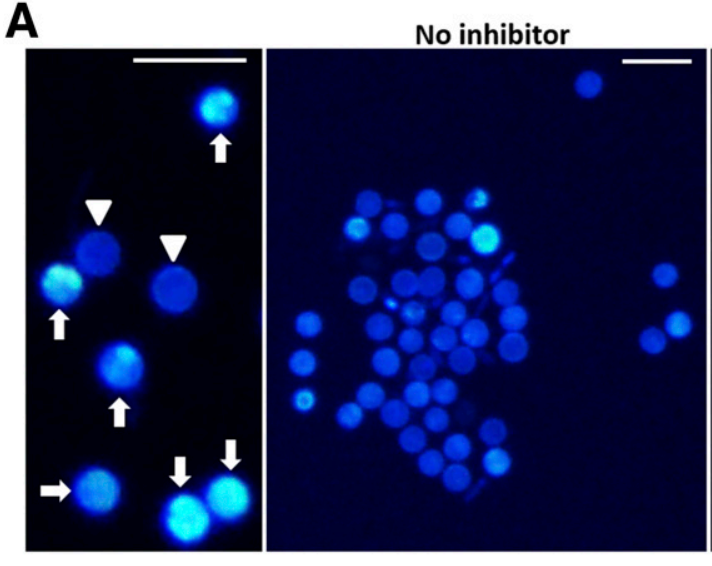

Inhibitor-infected group A
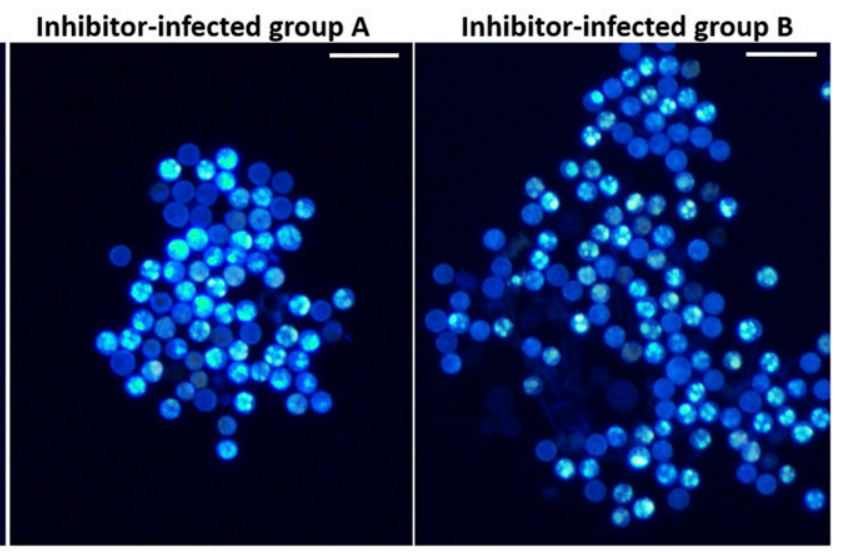

B

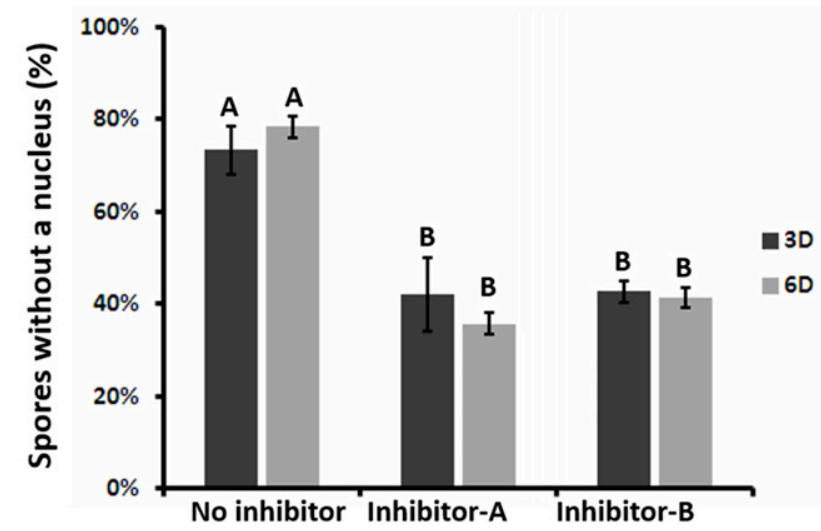

Fig. 4. Effect of mitogen-activated protein kinase kinase (MAPKK) inhibitor U0126 on resting spores of Plasmodiophora brassicae germination. A, Resting spores of P. brassicae stained with 4'-6-diamidino-2-phenylindole-dihydrochloride (DAPI). Resting spores with (arrowheads) or without (arrows) a nucleus were observed under UV excitation. Images show resting spores inoculated with root exudates with or without MAPKK inhibitor U0126 treatment for 6 days. The concentration for inhibitor-treated group A is $50 \mathrm{nM}$ and the concentration for inhibitor-treated group B is $100 \mathrm{nM}$. Scale bar $=10 \mu \mathrm{m}$. B, Mean percentage of $P$. brassicae resting spores without a nucleus stained with DAPI after incubation in MAPKK inhibitor U0126 treatment for 3 and 6 days. Error bars represent standard deviation values obtained from three biological replicates. Different letters indicate significant differences as determined by $t$ tests $(P \leq 0.05)$. 
plants treated without inhibitor, 42.3 in the inhibitor-treated group A, and 36 in inhibitor-treated group B. Using quantitative PCR, the relative accumulation amount of $P$. brassicae in the root tissues of host plants was evaluated. The density of $P$. brassicae in the roots of inhibitor-treated groups A and B was approximately 93 and $85 \%$ lower, respectively, than that in the roots of infected plants without inhibitor treatment; $P$. brassicae was not detected in the noninfected group (Fig. 5C). These results suggested that the pathogen density was significantly reduced by MAPKK inhibitor U0126 treatment. Taken together, our results showed that MAPKK-specific inhibitor U0126 could relieve the clubroot symptom of infected roots and reduce the pathogenicity, and indicated that MAPK pathways may play important roles during $P$. brassicae infection.

MAPKK inhibitor U0126 suppressed $P$. brassicae growth. Because MAPKK inhibitor U0126 led to reduced symptom development, we were interested in the extent to which the development of the MAPKK inhibitor was inhibited. The life cycle of $P$. brassicae can be divided into two phases: (i) the primary phase, which is restricted to the root hairs, and (ii) the secondary phase, during which the pathogen penetrates into the root cortex (Ingram and Tommerup 1972). The basic structures occurring during the secondary phase of the life cycle (young and vegetative plasmodia,

A Non-infected

Pathogen-infected, no inhibitor

inhibitor-treated group A

inhibitor-treated group B
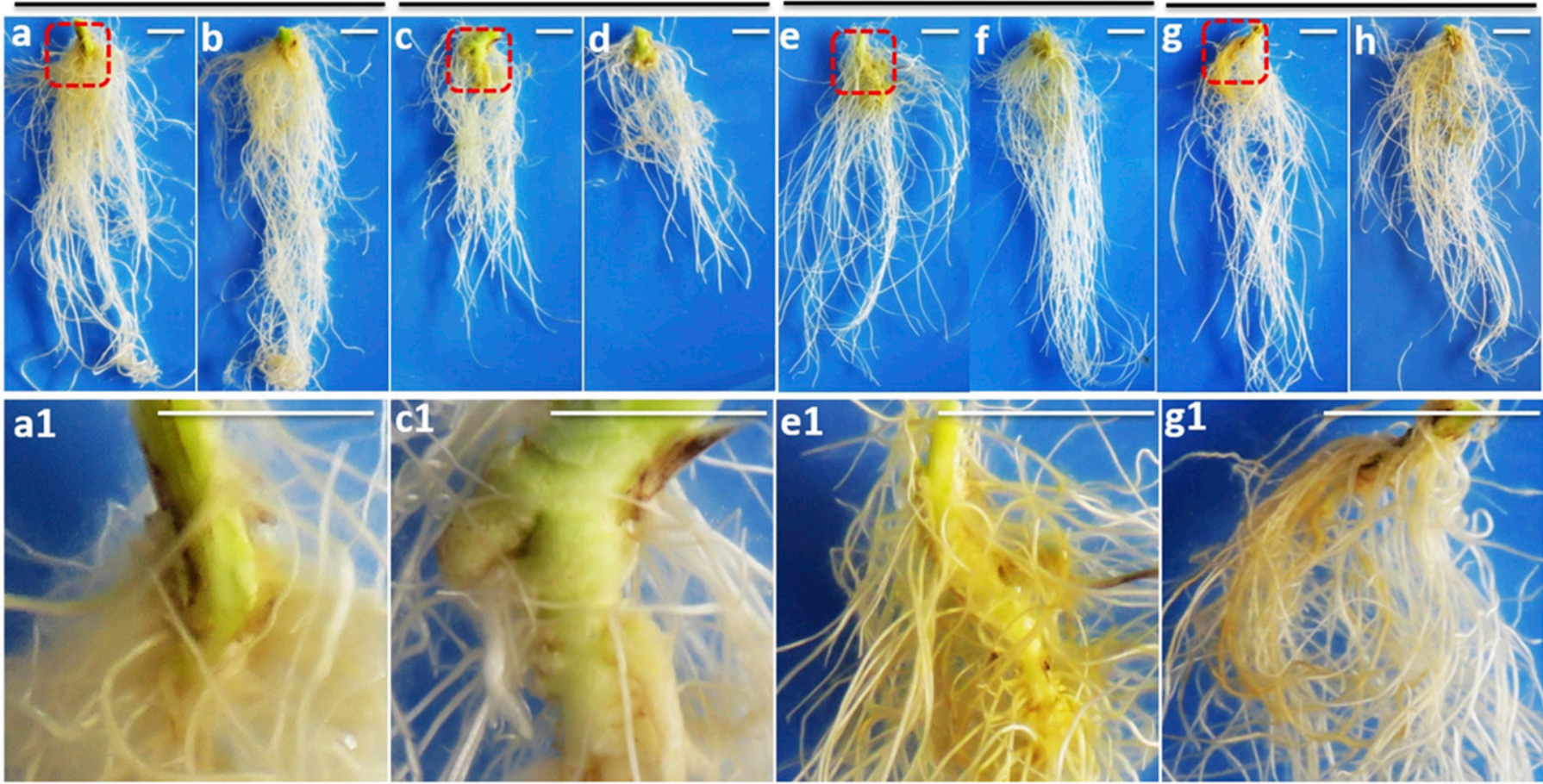

B DI: $\quad 87.5 \pm 3.7 \quad 42.3 \pm 6.3 \quad 36.0 \pm 4.9$
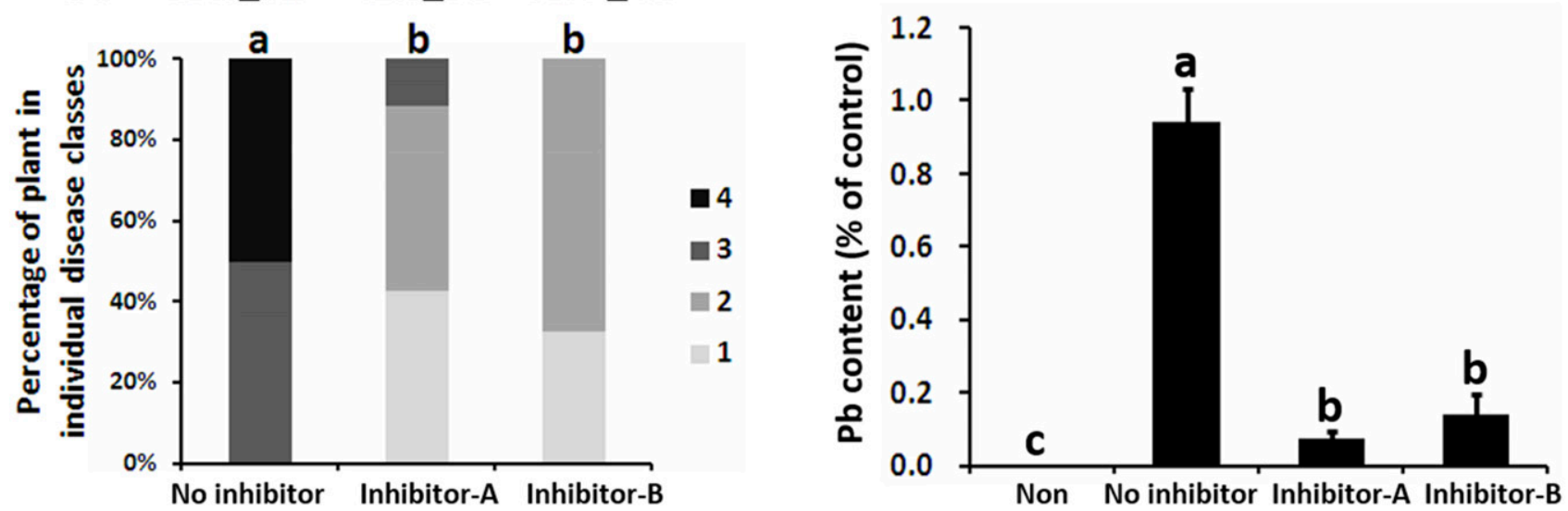

Fig. 5. Mitogen-activated protein kinase kinase (MAPKK) inhibitor U0126 treatment of rapeseed infected by Plasmodiophora brassicae. A, Gall phenotype of rapeseed infected with $P$. brassicae for 35 days in different treatments. Plant roots inoculated with neither P. brassicae nor MAPKK inhibitor U0126 (a and b, noninfected), inoculated with $P$. brassicae but not with MAPKK inhibitor U0126 (c and d, pathogen-infected group without inhibitor treatment), inoculated with P. brassicae and $50 \mathrm{nM}$ MAPKK inhibitor U0126 (e and f, inhibitor-treated group A), and inoculated with P. brassicae and $100 \mathrm{nM}$ MAPKK inhibitor U0126 ( $\mathrm{g}$ and $\mathrm{h}$, inhibitor-treated group B). Photos a1, c1, e1, and $\mathrm{g} 1$ are enlarged pictures of the dotted boxes in photos a, c, e, and g, respectively. B, Phytopathological analysis of rapeseed inoculated with $P$. brassicae and MAPKK inhibitor U0126. Percentages of plants in the individual disease classes are shown; DI $=$ disease index. Scale: $0=$ no symptoms; $1=$ very small galls mainly on lateral roots and that do not impair the main root; $2=$ small galls covering the main root and a few lateral roots; $3=$ medium to large galls on both lateral roots and main root; and $4=$ severe galls on lateral root, main root, or rosette, fine roots completely destroyed. For each treatment, 22 to 25 rapeseed plants were analyzed. Error bars represent standard deviation (SD) values obtained from three biological replicates. Different letters indicate significant differences as determined by $t$ tests $(P \leq 0.05)$. C, Pathogen DNA quantification $(\mathrm{Pb})$ by quantitative polymerase chain reaction. Results are expressed as the percentage of the mean $\mathrm{Pb}$ content in inoculated roots at 35 days postinoculation with $P$. brassicae and with or without MAPKK inhibitor U0126 treatment. At least three plant roots were taken as a mixed sample. Error bars represent SD values obtained from three biological replicates. Different letters indicate significant differences as determined by $t$ tests $(P \leq 0.05)$. 
sporangia, and RS) have been previously identified by scanning electron microscopy (Ludwig-Müller et al. 1999). The development of infection was examined in the inhibitor U0126 treatment and control groups at 35 dpi (Fig. 6). There were no zoospores in the negative control (Fig. 6A). Many mature RS were found in the root cells of $B$. rapa inoculated with $P$. brassicae (Fig. 6B), while developing vegetative plasmodia were observed in the MAPKK inhibitor U0126 treatment group A and group B (Fig. 6C and D). These different observations suggested that MAPKK inhibitor could inhibit the development of $P$. brassicae.

\section{DISCUSSION}

MAPK act as central signaling hubs that influence a broad range of cellular processes such as proliferation, differentiation, migration, and apoptosis (Osaki and Gama 2013; Rumora and Grubišić 2008). MAPK pathways are conserved signal transduction modules composed of three sequentially activated kinases, including MAPK, MAPKK, and MAPKKK. The numbers of MAPK, MAPKK, and MAPKKK are different among eukaryotic organisms. The present study, for the first time, provides a full list of the MAPK, MAPKK, and MAPKKK in $P$. brassicae, an obligate biotroph protist in the plasmodiophorids within the eukaryote supergroup Rhizaria. A genome-wide search of $P$. brassicae protein database by BLASTP resulted in the identification of seven $P b M A P K$, three $P b M A P K K$, and nine PbMAPKKK genes in $P$. brassicae (Table 1). We characterized all of the members of MAPK cascades of $P$. brassicae and performed a phylogenetic analysis in comparison with the members of Arabidopsis MAPK cascade proteins. The conserved domains or motifs and phylogenetic relationships strongly support the identity of each group (Fig. 1). The expression of MAPK cascade genes in three different zoosporic stages was profiled, and the results showed that a majority of the genes were upregulated in the CI stage, especially PbMAPK genes (Fig. 2). Different expression levels of the members of PbMAPK, PbMAPKK, and PbMAPKKK families were found in different zoosporic development stages, suggesting their important roles in regulating $P$. brassicae growth and development (Fig. 2). In addition, yeast two-hybrid assays led to the identification of three pairs of interacting partners: $P b M A P K K K 7$ and $P b M A P K K 3, P b M A P K K 3$ and PbMAPK1, and PbMAPKK3 and $P b M A P K 3$ (Fig. 3). These results suggest the existence of a likely linear pathway of PbMAPKKK7-PbMAPKK3-PbMAPK1/PbMAPK3. Further experiments could be carried out on other systems to accurately determine the linear modules involved in the signaling pathway. Due to the complexity of MAPK cascade, we did not identify all of the MAPKKK-MAPKK and MAPKK-MAPK interaction pairs. In addition, it is difficult to clone genes from $P$. brassicae for its high GC-content (58.5\%) (Schwelm et al. 2015). Furthermore, MAPK signaling cascades form complex interconnected networks within cells (Pedley and Martin 2005). Only a few MAPK cascade components have been studied in detail because their biological functions are often intertwined, redundant, and pleiotropic (Friedman and Perrimon 2006). After the characterization of the first MAPK from a fungal plant pathogen (Xu and Hamer 1996), similar strategies have been employed to examine the roles of MAPK in other phytopathogenic fungi. Generally, degenerate primers allow PCR-based amplification of MAPK genes, and the associated functions can be assessed through loss-of-function studies. For instance, knockout strains can be examined for phenotypes of vegetative growth and virulence. Due to the feature of obligate biotroph protist, no stable and successful genetic transformation system for $P$. brassicae is available to date, making it hard to identify its potential biological roles in P. brassicae.

MAPK signal transduction pathways have been reported in the pathogenesis of diverse plant pathogens (Hamel et al. 2012; Zhao et al. 2007). Kss1-2 (MAPK) of U. maydis participates in the regulation of virulence because the deletion mutants were unable to penetrate the plant cuticle (Brachmann et al. 2003), and Kss1-1/Kss1-2 double knockouts failed to mate and were nonpathogenic (Brefort et al. 2009). The PMK1 (MAPK) kinase is essential for appressorium formation

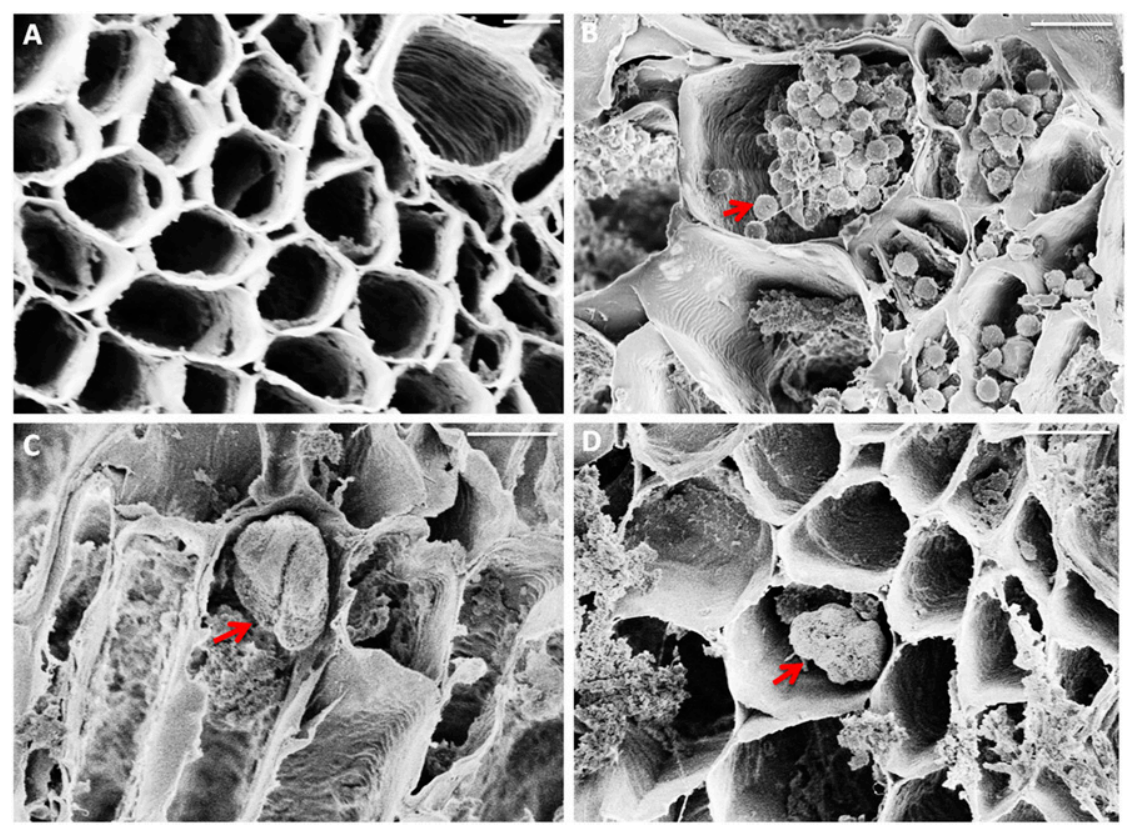

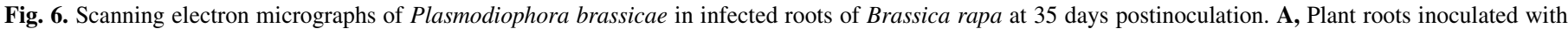

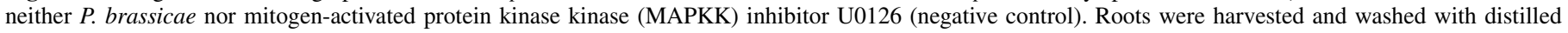

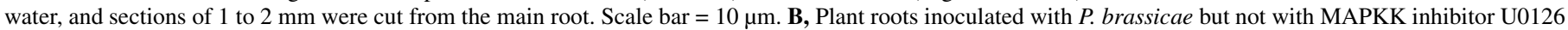

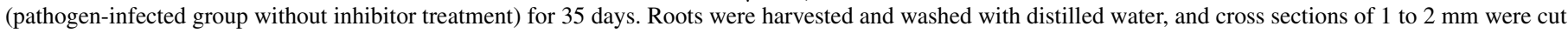

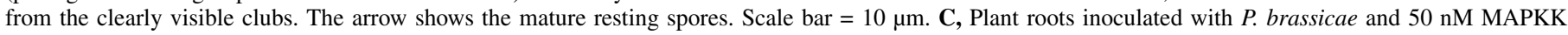

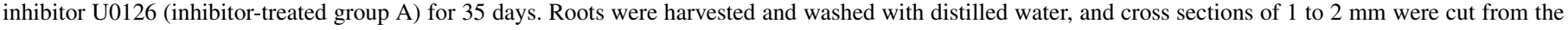

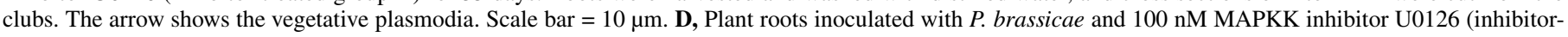

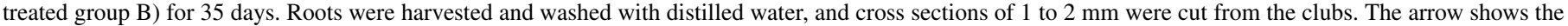
vegetative plasmodia. Scale bar $=10 \mu \mathrm{m}$. 
and the invasive growth of $M$. grisea (Xu 2000). MAPKKK Mst11 and MAPKK Mst7 are the upstream components of the PMK1 pathway, and the $m s t 7$ and $m s t 11$ mutants failed to form appressoria and were nonpathogenic (Zhao et al. 2005). Human p38 MAPK inhibitors reduced in vitro and in vivo the replication of the protozoan parasites Toxoplasma gondii, Encephalitozoon cuniculi, and Plasmodium falciparum (Brumlik et al. 2011; Wei et al. 2002, 2007). These findings suggest that p38 MAPK plays essential roles in the plasmodial life cycle by suppressing the development of plasmodial sp. U0126, a potent and specific inhibitor of MEK1 and MEK2 but with little effect on other kinases such as Cdk2, ERK, JNK, MEKK, and Raf (Favata et al. 1998). MAPKK inhibitor U0126 was reported to affect the meiotic cell cycle progression in mouse oocytes, including microtuble organization, asymmetric division, and metaphase II arrest (Tong et al. 2003). The Ras/Raf/Mek/Erk intracellular signaling pathway connects cell surface receptors to gene transcription factors, which regulate gene expression to control cellular proliferation, cell differentiation, and apoptosis. This pathway is erroneously activated in many types of cancers, and is thought to play a crucial role in tumorogenesis. The growth of cancer cell lines HepG2, MiaPaca, Panc-1, and HT-29 all showed a significant decrease after treatment with MAPKK inhibitor U0126 (Mackin et al. 2006). In this study, we also found that U0126 significantly inhibited the germination of $P$. brassicae RS (Fig. 4). In addition, MAPKK inhibitor U0126 treatment relieved the severity of clubroot symptoms (Fig. 5A), resulting in reduced disease index (Fig. 5B). Moreover, a low disease index was correlated with smaller amounts of PbActin transcripts (Fig. 5C). In addition, the growth and development of $P$. brassicae were decreased (Fig. 6). Both microscopic and molecular analysis indicate that MAPKK inhibitor U0126 treatment delays the life cycle of $P$. brassicae and, in particular, delays the production of RS. We could still detect the development of P. brassicae under inhibitor U0126 treatments. These results suggested that U0126 did not kill all of the RS but only a very small amount of RS germinated and developed vegetative plasmodia. However, the number of plasmodia is too few and that has little effect on the growth of plants. It is not clear yet whether MAPKK inhibitor U0126 is targeted to host plant MAPKK. U0126 inhibited root hair growth in alfalfa, induced vacuolation and swelling of growing root hairs, and root development was slightly impaired by U0126 treatment in Medicago truncatula (Ryu et al. 2017; Šmaj et al. 2002). The concentration of U0126 used in this study was 100 -fold lower than the treatment of M. truncatula (Ryu et al. 2017; Šamaj et al. 2002), and the growing root hairs were not affected by treatment with MAPKK inhibitor U0126 (Supplementary Fig. S5). The root and shoot development appeared to be normal (Fig. $5 \mathrm{~A}$ ) and the root length was not affected by treatment with MAPKK inhibitor U0126. Because the concentrations of the MAPKK inhibitor required to reduce the pathogenicity of $P$. brassicae are not toxic for the host cells, our findings may be important with respect to plasmodial drug development. Therefore, the results of the present study provide a significant foundation for further investigating the biological functions of MAPK cascades and pathogenic mechanisms of $P$. brassicae and other plasmodiophorid pathogens, and inhibitor U0126 could be used for management of clubroot disease.

\section{ACKNOWLEDGMENTS}

We thank D. Gao, B.-C. Xu, and P. Zhang of the core facility in the Wuhan Institute of Virology for their technical support in scanning electron microscopy.

\section{LITERATURE CITED}

Allen, G. C., Flores-Vergara, M. A., Krasnyanski, S., Kumar, S., and Thompson, W. F. 2006. A modified protocol for rapid DNA isolation from plant tissues using cetyltrimethylammonium bromide. Nat. Protoc. 1: 2320-2325.

Andrusiak, M. G., and Jin, Y. 2016. Context specificity of stress-activated mitogen-activated protein (MAP) kinase signaling: The story as told by Caenorhabditis elegans. J. Biol. Chem. 291:7796-7804.
Aouadi, M., Binetruy, B., Caron, L., Marchand-Brustel, Y. L., and Bost, F. 2006. Role of MAPKs in development and differentiation: Lessons from knockout mice. Biochimie 88:1091-1098.

Asai, T., Tena, G., Plotnikova, J., Willmann, M. R., Chiu, W. L., Gomez-Gomez, L., Boller, T., Ausubel, F. M., and Sheen, J. 2002. MAP kinase signalling cascade in Arabidopsis innate immunity. Nature 415:977-983.

Bailey, T. L., Williams, N., Misleh, C., and Li, W. W. 2006. MEME: Discovering and analyzing DNA and protein sequence motifs. Nucleic Acids Res. 34:W369-W373.

Bi, K., He, Z., Gao, Z., Zhao, Y., Fu, Y., Cheng, J., Xie, J., Jiang, D., and Chen, T. 2016. Integrated omics study of lipid droplets from Plasmodiophora brassicae. Sci. Rep. 6: Article 36965.

Brachmann, A., Schirawski, J., Müller, P., and Kahmann, R. 2003. An unusual MAP kinase is required for efficient penetration of the plant surface by Ustilago maydis. EMBO J. 22:2199-2210.

Braselton, J. P. 1995. Current status of the plasmodiophorids. Crit. Rev. Microbiol. 21:263-275.

Brefort, T., Doehlemann, G., Mendoza-Mendoza, A., Reissmann, S., Djamei, A., and Kahmann, R. 2009. Ustilago maydis as a Pathogen. Annu. Rev. Phytopathol. 47:423-445.

Brumlik, M. J., Nkhoma, S., Kious, M. J., Iii, G. R. T., Patterson, T. F., Siekierka, J. J., Anderson, T. J. C., and Curiel, T. J. 2011. Human p38 mitogen-activated protein kinase inhibitor drugs inhibit Plasmodium falciparum replication. Exp. Parasitol. 128:170-175.

Chang, L., and Karin, M. 2001. Mammalian MAP kinase signalling cascades. Nature 410:37-40.

Chen, R. E., and Thorner, J. 2007. Function and regulation in MAPK signaling pathways: Lessons learned from the yeast Saccharomyces cerevisiae. Biochim. Biophys. Acta 1773:1311-1340.

Chen, T., Bi, K., He, Z., Gao, Z., Zhao, Y., Fu, Y., Cheng, J., Xie, J., and Jiang, D. 2016. Arabidopsis mutant bikl exhibits strong resistance to Plasmodiophora brassicae. Front. Physiol. 7:402.

Dixon, G. R. 2009. The occurrence and economic impact of Plasmodiophora brassicae and Clubroot disease. J. Plant Growth Regul. 28:194-202.

Favata, M. F., Horiuchi, K. Y., Manos, E. J., Daulerio, A. J., Stradley, D. A., Feeser, W. S., Van Dyk, D. E., Pitts, W. J., Earl, R. A., and Hobbs, F. 1998. Identification of a novel inhibitor of mitogen-activated protein kinase kinase. J. Biol. Chem. 273:18623-18632.

Friedman, A., and Perrimon, N. 2006. High-throughput approaches to dissecting MAPK signaling pathways. Methods 40:262-271.

Gao, M. H., Liu, J. M., Bi, D. L., Zhang, Z. B., Cheng, F., Chen, S. F., and Zhang, Y. L. 2008. MEKK1, MKK1/MKK2 and MPK4 function together in a mitogen-activated protein kinase cascade to regulate innate immunity in plants. Cell Res. 18:1190-1198.

Hamel, L.-P., Nicole, M.-C., Duplessis, S., and Ellis, B. E. 2012. Mitogenactivated protein kinase signaling in plant-interacting fungi: Distinct messages from conserved messengers. Plant Cell 24:1327-1351.

Hwang, S. F., Strelkov, S. E., Feng, J., Gossen, B. D., and Howard, R. J. 2012. Plasmodiophora brassicae: A review of an emerging pathogen of the Canadian canola (Brassica napus) crop. Mol. Plant Pathol. 13:105-113.

Ichimura, K., Shinozaki, K., Tena, G., Sheen, J., Henry, Y., Champion, A., Kreis, M., Zhang, S. Q., Hirt, H., Wilson, C., Heberle-Bors, E., Ellis, B. E., Morris, P. C., Innes, R.W., Ecker, J. R., Scheel, D., Klessig, D. F., Machida, Y., Mundy, J., Ohashi, Y., and Walker, J. C. 2002. Mitogen-activated protein kinase cascades in plants: A new nomenclature. Trends Plant Sci. 7: 301-308.

Ingram, D. S., and Tommerup, I. C. 1972. The life history of Plasmodiophora brassicae Woron. R. Soc. Lond. Proc. Ser. B Biol. Sci. 180:103-112.

Jäschke, D., Dugassa-Gobena, D., Karlovsky, P., Vidal, S., and Ludwig-Müller, J. 2010. Suppression of clubroot (Plasmodiophora brassicae) development in Arabidopsis thaliana by the endophytic fungus Acremonium alternatum. Plant Pathol. 59:100-111.

Jonak, C., Okrész, L., Bögre, L., and Hirt, H. 2002. Complexity, cross talk and integration of plant MAP kinase signalling. Curr. Opin. Plant Biol. 5:415-424.

Kageyama, K., and Asano, T. 2009. Life cycle of Plasmodiophora brassicae. J. Plant Growth Regul. 28:203-211.

Katz, M., Amit, I., and Yarden, Y. 2007. Regulation of MAPKs by growth factors and receptor tyrosine kinases. Biochim. Biophys. Acta 1773:1161-1176.

Ludwig-Müller, J., Bennett, R. N., Kiddle, G., Ihmig, S., Ruppel, M., and Hilgenberg, W. 1999. The host range of Plasmodiophora brassicae and its relationship to endogenous glucosinolate content. New Phytol. 141:443-458.

Mackin, K. M., Deming, D. A., Geiger, P., Chen, H., and Holen, K. 2006. Effects of the Mek inhibitor UO126 on growth of cancer cell lines HepG2, MiaPaca, Panc-1, and HT-29. J. Surg. Res. 130:262.

Marchler-Bauer, A., Lu, S., Anderson, J. B., Chitsaz, F., Derbyshire, M. K., DeWeese-Scott, C., Fong, J. H., Geer, L. Y., Geer, R. C., Gonzales, N. R., Gwadz, M., Hurwitz, D. I., Jackson, J. D., Ke, Z., Lanczycki, C. J., Lu, F., Marchler, G. H., Mullokandov, M., Omelchenko, M. V., Robertson, C. L., Song, J. S., Thanki, N., Yamashita, R. A., Zhang, D., Zhang, N., Zheng, C., 
and Bryant, S. H. 2011. CDD: A Conserved Domain Database for the functional annotation of proteins. Nucleic Acids Res. 39:D225-D229.

Meng, X., and Zhang, S. 2013. MAPK cascades in plant disease resistance signaling. Annu. Rev. Phytopathol. 51:245-266.

Niwa, R., Nomura, Y., Osaki, M., and Ezawa, T. 2008. Suppression of clubroot disease under neutral $\mathrm{pH}$ caused by inhibition of spore germination of Plasmodiophora brassicae in the rhizosphere. Plant Pathol. 57:445-452.

Osaki, L. H., and Gama, P. 2013. MAPKs and signal transduction in the control of gastrointestinal epithelial cell proliferation and differentiation. Int. J. Mol. Sci. 14:10143-10161.

Pedley, K. F., and Martin, G. B. 2005. Role of mitogen-activated protein kinases in plant immunity. Curr. Opin. Plant Biol. 8:541-547.

Plowman, G. D., Sudarsanam, S., Bingham, J., Whyte, D., and Hunter, T. 1999. The protein kinases of Caenorhabditis elegans: A model for signal transduction in multicellular organisms. Proc. Natl. Acad. Sci. USA 96:13603-13610.

Rao, K. P., Richa, T., Kumar, K., Raghuram, B., and Sinha, A. K. 2010. In silico analysis reveals 75 members of mitogen-activated protein kinase kinase kinase gene family in rice. DNA Res. 17:139-153.

Rolfe, S. A., Strelkov, S. E., Links, M. G., Clarke, W. E., Robinson, S. J., Djavaheri, M., Malinowski, R., Haddadi, P., Kagale, S., and Parkin, I. A. P. 2016. The compact genome of the plant pathogen Plasmodiophora brassicae is adapted to intracellular interactions with host Brassica spp. BMC Genomics 17:272.

Rumora, L., and Grubišić, T. Ž. 2008. MAP kinase signalling cascades in cell proliferation and apoptosis. Research Signpost 151-171.

Ryu, H., Laffont, C., Frugier, F., and Hwang, I. 2017. MAP kinase-mediated negative regulation of symbiotic nodule formation in Medicago truncatula. Mol. Cells 40:17-23.

Saitou, N., and Nei, M. 1987. The neighbor-joining method: A new method for reconstructing phylogenetic trees. Mol. Biol. Evol. 4:406.

Šamaj, J., Ovecka, M., Hlavacka, A., Lecourieux, F., Meskiene, I., Lichtscheidl, I., Lenart, P., Salaj, J., Volkmann, D., and Bögre, L. 2002. Involvement of the mitogen-activated protein kinase SIMK in regulation of root hair tip growth. EMBO J. 21:3296-3306.

Schwelm, A., Fogelqvist, J., Knaust, A., Julke, S., Lilja, T., Bonilla-Rosso, G., Karlsson, M., Shevchenko, A., Dhandapani, V., Choi, S. R., Kim, H. G., Park, J. Y., Lim, Y. P., Ludwig-Müller, J., and Dixelius, C. 2015. The Plasmodiophora brassicae genome reveals insights in its life cycle and ancestry of chitin synthases. Sci. Rep. 5:11153.

Siemens, J., Nagel, M., Ludwig-Müller, J., and Sacristan, M. D. 2002. The interaction of Plasmodiophora brassicae and Arabidopsis thaliana: $\mathrm{Pa}-$ rameters for disease quantification and screening of mutant lines. J. Phytopathol. 150:592-605.
Singh, R., Lee, M. O., Lee, J. E., Choi, J., Ji, H. P., Kim, E. H., Ran, H. Y., Cho, J. I., Jeon, J. S., and Rakwal, R. 2012. Rice mitogen-activated protein kinase interactome analysis using the yeast two-hybrid system. Plant Physiol. 160:477-487.

Tong, C., Fan, H. Y., Chen, D. Y., Song, X. F., Schatten, H., and Sun, Q. Y. 2003. Effects of MEK inhibitor U0126 on meiotic progression in mouse oocytes: Microtuble organization, asymmetric division and metaphase II arrest. Cell Res. 13:375-383.

Wang, H. C., Ngwenyama, N., Liu, Y. D., Walker, J. C., and Zhang, S. Q. 2007. Stomatal development and patterning are regulated by environmentally responsive mitogen-activated protein kinases in Arabidopsis. Plant Cell 19:63-73.

Wang, J., Pan, C., Wang, Y., Ye, L., Wu, J., Chen, L., Zou, T., and Lu, G. 2015. Genome-wide identification of MAPK, MAPKK, and MAPKKK gene families and transcriptional profiling analysis during development and stress response in cucumber. BMC Genomics 16:386.

Wei, S., Daniel, B. J., Brumlik, M. J., Burow, M. E., Zou, W., Khan, I. A., Wadsworth, S., Siekierka, J., and Curiel, T. J. 2007. Drugs designed to inhibit human p38 mitogen-activated protein kinase activation treat Toxoplasma gondii and Encephalitozoon cuniculi infection. Antimicrob. Agents Chemother. 51:4324-4328.

Wei, S., Marches, F., Daniel, B., Sonda, S., Heidenreich, K., and Curiel, T. 2002. Pyridinylimidazole p38 mitogen-activated protein kinase inhibitors block intracellular Toxoplasma gondii replication. Int. J. Parasitol. 32: 969-977.

Xia, Z., Dickens, M., Raingeaud, J., Davis, R. J., and Greenberg, M. E. 1995. Opposing effects of ERK and JNK-p38 MAP kinases on apoptosis. Science 270:1326-1331.

$\mathrm{Xu}$, J. R. 2000. Map kinases in fungal pathogens. Fungal Genet. Biol. 31: $137-152$.

$\mathrm{Xu}$, J. R., and Hamer, J. E. 1996. MAP kinase and cAMP signaling regulate infection structure formation and pathogenic growth in the rice blast fungus Magnaporthe grisea. Genes Dev. 10:2696-2706.

Yu, C.-S., Chen, Y.-C., Lu, C.-H., and Hwang, J.-K. 2006. Prediction of protein subcellular localization. Proteins 64:643-651.

Zhang, W., and Liu, H. T. 2002. MAPK signal pathways in the regulation of cell proliferation in mammalian cells. Cell Res. 12:9-18.

Zhao, X., Kim, Y., Park, G., and Xu, J.-R. 2005. A mitogen-activated protein kinase cascade regulating infection-related morphogenesis in Magnaporthe grisea. Plant Cell 17:1317-1329.

Zhao, X., Mehrabi, R., and Xu, J. R. 2007. Mitogen-activated protein kinase pathways and fungal pathogenesis. Eukaryot. Cell 6:1701-1714. 